\title{
金属-有机骨架材料中吸附气体的扩散速率
}

\author{
穆鞾刘大欢* 阳庆元仲崇立 \\ (北京化工大学化学工程学院计算化学研究室, 北京 100029)
}

\begin{abstract}
摘要：采用分子动力学方法, 以甲烷为探针分子研究了不同压力条件下气体在具有不同孔道结构的金属-有机 骨架材料(MOFs)中的扩散速率. 通过计算气体在八种材料中的自扩散系数, 并结合气体分子在材料中的质心分 布图等, 讨论了气体扩散速率与孔道结构之间的关系. 研究结果表明: 对于同时含有孔笼(pocket)和三维正交孔 道(channel)结构的 MOF 材料(P-C 材料), 低压时甲烷气体吸附在孔笼结构中, 随着压力的升高, 气体分子开始进 人正交孔道, 同时其自扩散系数增加; 而对于只含有三维立方孔道结构的 IRMOF(isoreticular MOF)系列材料, 在 中低压范围内, 气体分子在其中的自扩散系数随压力变化较小. 当压力进一步升高时, 气体分子在材料孔道中的 吸附逐渐接近饱和, 其自扩散系数均降低. 因此, 在不同 MOF 材料中气体分子扩散速率的差异主要取决于孔道 结构的不同. 对 P-C 材料, 中低压下通过控制压力可以控制气体在其中的扩散速率, 从而为 MOF 材料在气体存 储、分离等方面的实际应用提供参考信息.
\end{abstract}

关键词：分子模拟；金属-有机骨架材料；扩散；化工应用

中图分类号：0641

\section{Diffusion Rates of Gas Molecules Adsorbed in Metal-Organic Frameworks}

\author{
MU Wei LIU Da-Huan* YANG Qing-Yuan ZHONG Chong-Li \\ (Laboratory of Computational Chemistry, College of Chemical Engineering, \\ Beijing University of Chemical Technology, Beijing 100029, P. R. China)
}

\begin{abstract}
A systematic equilibrium molecular dynamics study was performed to investigate the diffusion rates of gas molecules as a function of the pressure in metal-organic frameworks (MOFs) with different structures. Methane was chosen as the probe molecule. The self-diffusion coefficients in eight typical MOFs were calculated at room temperature. Combined self-diffusion coefficients with the contour plots of the center of mass (COM) probability densities of methane, the relationship between the diffusion rates of gas molecules and the structure of the pores in the MOFs is discussed. Results show that methane tends to adsorb in pockets in MOFs with pocket and channel pores (P-C materials) at low pressure. With an increase in pressure, the gas molecules move to the channel and the self-diffusion coefficient increases. However, the diffusion coefficient of methane changes a little in the low and middle pressure range in the IRMOFs (isoreticular MOFs) with only one kind of pore. With a further increase in pressure, the self-diffusion coefficient of methane decreases in all the studied MOFs. Therefore, the difference in diffusion rates of methane in different MOFs may be mainly attributed to the pore structures of the materials. In addition, diffusion rates of the gas molecules in the P-C materials could be controlled in a wide range by varying the pressure, providing useful information for the application of MOFs in gas storage and separation.
\end{abstract}

Key Words: Molecular simulation; Metal-organic frameworks; Diffusion; Chemical engineering application

Received: January 21, 2010; Revised: March 4, 2010; Published on Web: April 23, 2010.

*Corresponding author. Email: liudh@mail.buct.edu.cn; Tel: +86-10-64431705.

The project was supported by the National Outstanding Young Scientists Foundation of China (20725622) and National Natural Science Foundation of China (20876006, 20821004, 20906002, 20706002).

国家杰出青年科学基金(20725622), 国家自然科学基金(20876006, 20821004, 20906002, 20706002)资助项目

(C) Editorial office of Acta Physico-Chimica Sinica 
金属-有机骨架材料(MOFs)因具有比表面积和 孔隙率大, 结构组成多样及热稳定性好等特点, 已成 为当前新功能材料研究的前沿和热点之一, 并在储 气、分离、催化、生物化学及制药等领域显示了广泛 的应用前景 ${ }^{[1]}$. 在分子水平上理解气体分子在 $\mathrm{MOF}$ 材料中的扩散行为与其结构特性之间的关系, 可以 为 $\mathrm{MOF}$ 材料的分子设计和工艺开发提供科学依据 与技术支持. 但由于 MOF 材料的结构非常复杂, 采 用实验的方法表征气体在 MOF 材料中的扩散行为 非常困难, 同时用不同实验手段测定出的结果差别 较大 ${ }^{[2]}$, 因此目前人们对 MOF 材料中的分子扩散行 为知之甚少, 而与此有关的信息几乎均来自于分子 动力学 (molecular dynamic, MD)模拟的计算结果 ${ }^{[3-9]}$. 研究人员利用分子模拟技术对一些纯气体在MOF 材料中的扩散行为进行了研究 ${ }^{[3-7]}$, 发现气体分子 在一些 MOF 材料中的扩散速率与其在沸石中相 当 ${ }^{[10-11]}$, 有希望在实际生产中得到应用, 因此, 此方 面的深人探讨可以为 MOF 材料在气体捕集、分离 等方面的应用提供理论依据. 但目前, 有关气体分 子的扩散速率与材料结构特征之间关系的工作十分 有限. Liu 等人 ${ }^{[12]}$ 利用分子模拟与动力学修正过渡态 理论 (dynamically corrected transition-state theory, dcTST)相结合的方法, 只给出了气体扩散势垒(diffusion barrier)的数量和强弱与 MOF 材料结构之间的 关系. 因此, 本工作主要对气体分子在具有不同结构 的 MOF 材料中的扩散行为进行系统研究, 重点讨 论扩散速率与 MOF 材料结构特征之间的关系, 希 望可以找到材料结构与扩散速率之间的联系, 为材 料的实际应用及新材料的设计提供帮助.

\section{1 模型与计算方法}

\section{1 模 型}

在 MOF 材料中存在两类具有独特结构特征的 材料: 一类是 IRMOF 材料, 是由 $\left[\mathrm{Zn}_{4} \mathrm{O}\right]^{6+}$ 无机基团与 有机配体(linker)桥联而成的三维立方微孔骨架; 另 一类材料是 pocket-channel (P-C) 材料, 具有与 $\mathrm{Cu}-$ BTC (均苯三甲酸) 类似的结构特性, 由 paddlewheel 结构的铜二聚体 $\mathrm{Cu}_{2}(\mathrm{COO})_{4}$ 与有机配体桥联 而成, 骨架内具有方形主正交孔道以及孔笼. 两种不 同孔结构之间通过直径较小的窗孔相互贯通. $\mathrm{Cu}-$ BTC, PCN-6 ${ }^{\prime}$, mesoMOF-1 和 MOF-HTB' ${ }^{\prime}$ 四种材料 属于 P-C 材料, 因有机配体的不同, 材料的两类孔 道及窗孔的尺寸也存在差异. 不同的孔道种类使 P-
C 材料相对 IRMOFs 结构更复杂, 因此本工作将这 两类 MOF 材料选为研究对象, 进而讨论 MOF 材料 骨架结构对分子扩散的影响。

在本工作中, 对材料的模拟采用刚性结构模型, 利用 $X$ 射线衍射数据 ${ }^{[13-16]}$ 通过 Materials Visualizer 软件 ${ }^{[17]}$ 构建. 本工作中 IRMOF 系列材料, 金属簇为 $\mathrm{Zn}_{4} \mathrm{O}$ 单元, linker 单元为带有稠环的二酸. 在这类材 料中, 每个 $\mathrm{Zn}_{4} \mathrm{O}$ 单元与 6 个 linker 单元连接, 而每 个 linker 单元与 2 个 $\mathrm{Zn}_{4} \mathrm{O}$ 单元连接. 图 1 中所示为 各种材料 linker. 其中 IRMOF-11 和 IRMOF-13 是 拥有连锁结构的材料, 以 IRMOF-14 和 IMROF-13 为例说明, 如图 2 所示.

图 3 为各种 P-C 材料的 linker 结构. 在这类材 料中, 每个金属簇单元与 4 个linker连接, 每个linker 单元与 3 个金属簇单元连接(图 2). 图 4 以 $\mathrm{Cu}-\mathrm{BTC}$ 为例 (见图4(a)), P-C材料中都含有近八面体结构的 孔笼(见图 4(b)), 由 4 个均苯三甲酸 $(\mathrm{Cu}-\mathrm{BTC}$ 中 linker 为 BTC)通过 6 个金属簇单元连接而成, 孔笼 相互连接构成含有空腔的 Cu-BTC 晶体. 每个孔笼 含有四个窗口孔道, 连接到正交孔道. 除与孔笼连接 之外, 正交孔道之间也相互连接, 连接口大致呈正方 形, 由于四种材料 linker 的不同, 正交孔道尺寸也不 相同: linker 尺寸越大, 正方形连接口越大.

\section{2 力场参数}

TraPPE 力场 ${ }^{[18]}$ 已被广泛应用于研究甲烷在介 孔材料中的吸附与扩散性质 ${ }^{[5,19]}$, 因此, 本工作中甲 烷分子由 TraPPE 力场描述, 即将甲烷分子看作一 个无电荷的单一作用点. MOF 材料的骨架原子采用 全原子(all-atom)模型的通用力场(UFF)参数, 此力场 被广泛应用于 MOF 材料中甲烷的模拟研究 ${ }^{[3,5,20]}$. 力 场中的势能参数见表 1 . 甲烷分子与各种材料之间 的相互作用由 Lennard-Jones (L-J) 12-6 势能模型描
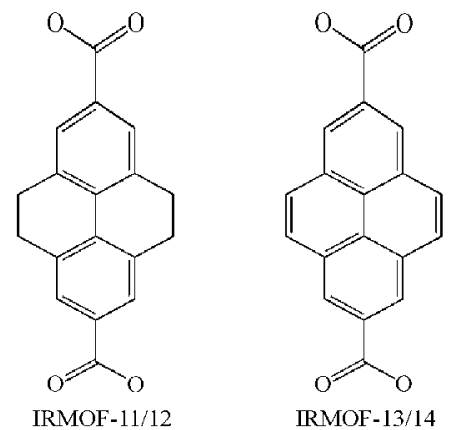

图 1 IRMOF 材料中 linker 结构

Fig.1 Linker structures in IRMOF materials 


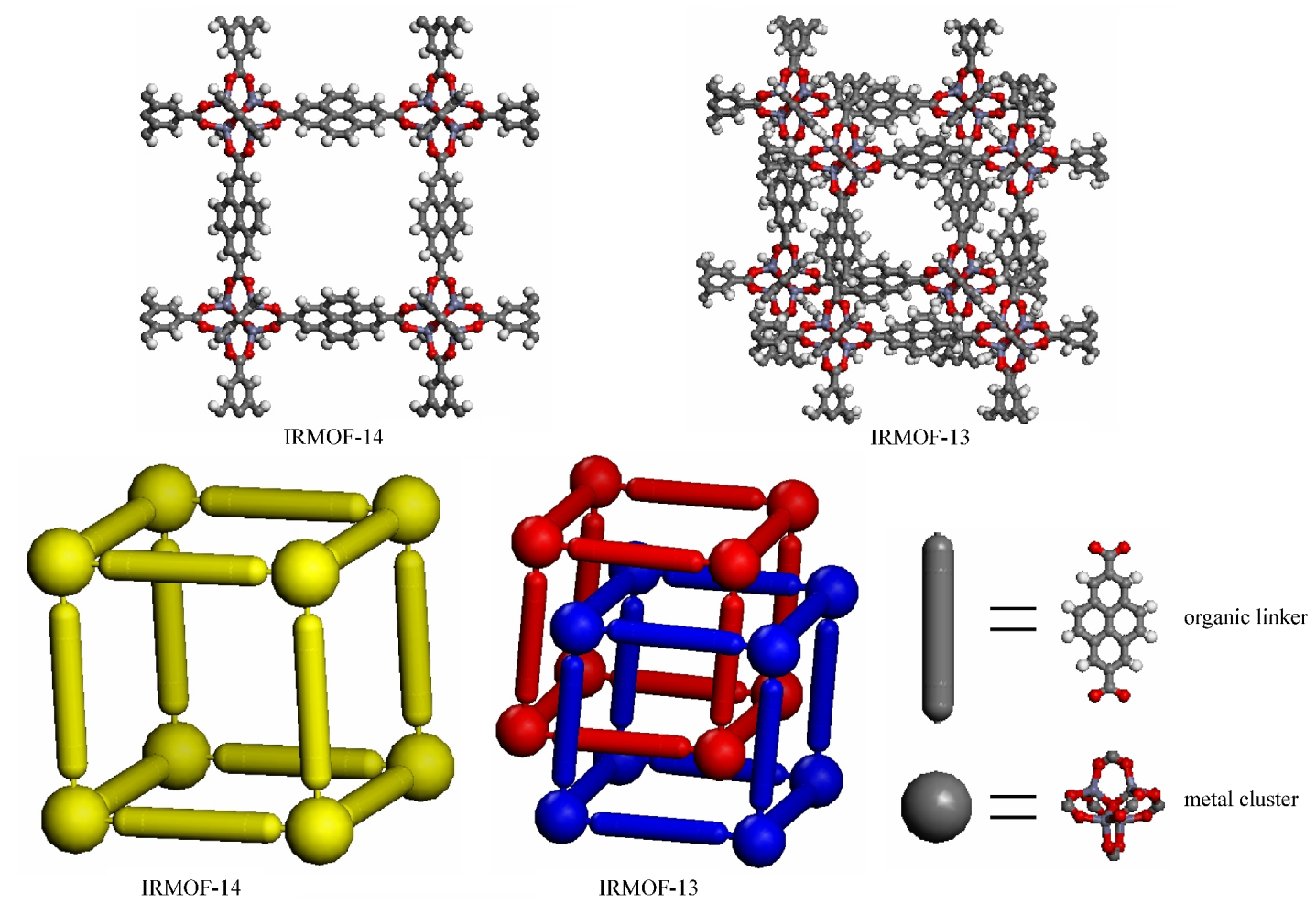

图 2 IRMOF-14 与 IRMOF-13 晶体结构单元

Fig.2 Unit cells of IRMOF-14 and IRMOF-13 crystal structures

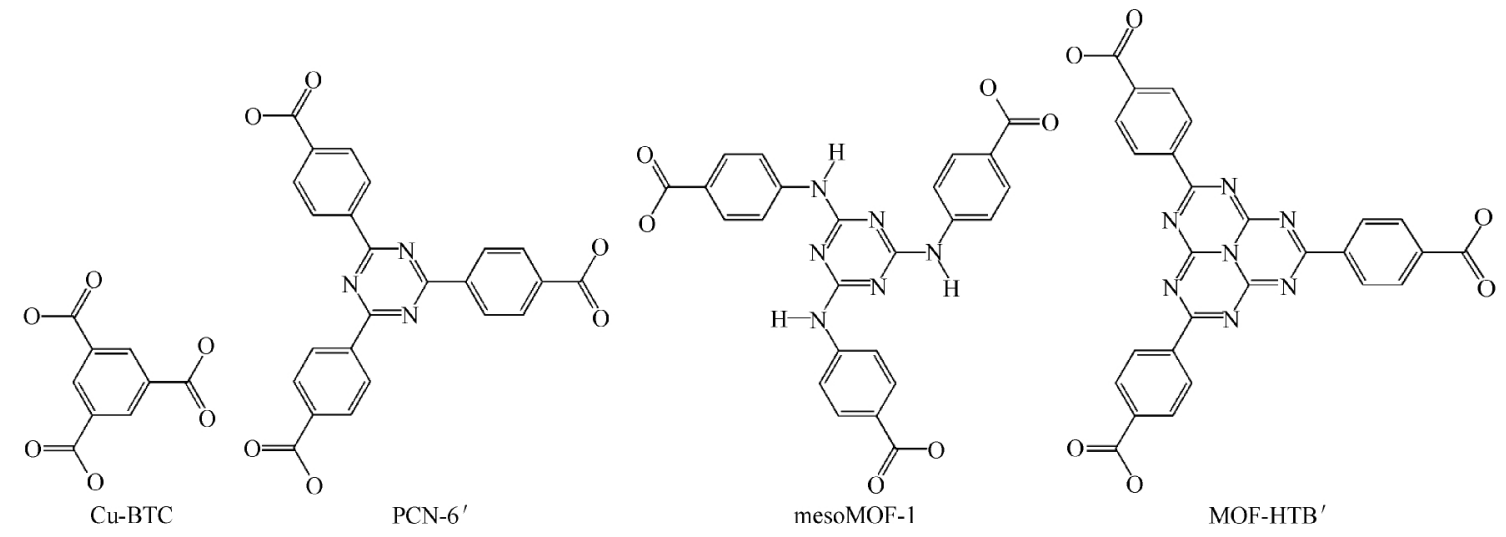

图 3 Pocket-channel 材料中 linker 结构单元

Fig.3 Linker structures in pocket-channel materials
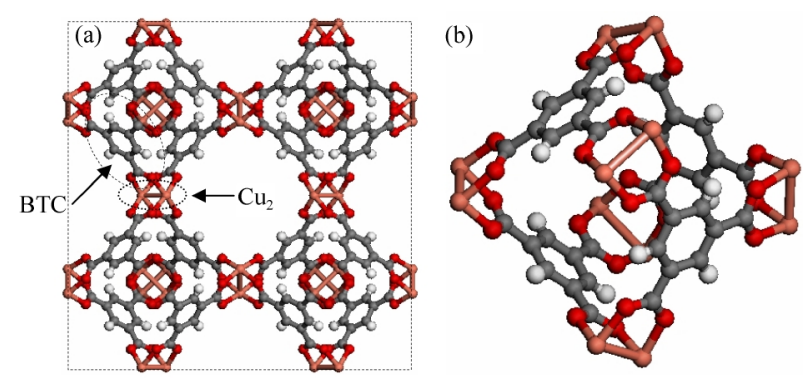

图 4 Cu-BTC 晶体结构

Fig.4 Crystal structure of $\mathrm{Cu}-\mathrm{BTC}$ (a) unit cell of $\mathrm{Cu}-\mathrm{BTC}$, (b) structure of pocket in $\mathrm{Cu}-\mathrm{BTC}$
述. L-J 作用点的交互作用参数可以通过 LorentzBerthelot 混合规则计算获得.

\section{3 计算方法}

采用分子动力学模拟方法, 对甲烷单组分体系 在 MOFs 中的扩散性质进行研究, 系综为 NVT 系 综. 为保证模拟精确, 模拟盒子中需存在足够多的气 体分子数, 所以对单元晶胞较小的 $\mathrm{Cu}-\mathrm{BTC}$ 等材料 选择 $2 \times 2 \times 2$ 个单元晶胞为模拟盒子. L-J 势能截断 半径取为 $1.7 \mathrm{~nm}$, 使用 Nosé-Hoover 恒温技术保持 温度在 $298 \mathrm{~K}$. 在进行分子动力学运算之前, 为得到 
表 1 本文中所使用甲烷及 MOF 材料的 L-J 势能参数

Table 1 L-J potential parameters for the framework atoms in MOFs and methane used in this work

\begin{tabular}{cccc}
\hline & \multicolumn{2}{c}{ L-J potential parameters } & \multirow{2}{*}{ Type of force field } \\
\cline { 2 - 3 } & $\sigma / \mathrm{nm}$ & $\left(\varepsilon k_{\mathrm{B}}^{-1}\right) / \mathrm{K}$ & \\
\hline $\mathrm{CH}_{4}$ & 0.3730 & 148.0 & TraPPE \\
$\mathrm{Cu}$ & 0.3114 & 2.517 & $\mathrm{UFF}$ \\
$\mathrm{Zn}$ & 0.2462 & 62.34 & $\mathrm{UFF}$ \\
$\mathrm{O}$ & 0.3118 & 30.21 & $\mathrm{UFF}$ \\
$\mathrm{C}$ & 0.3431 & 52.86 & $\mathrm{UFF}$ \\
$\mathrm{N}$ & 0.3261 & 34.69 & $\mathrm{UFF}$ \\
$\mathrm{H}$ & 0.2571 & 22.15 & UFF \\
\hline
\end{tabular}

不同压力下各种材料模拟盒子中甲烷的吸附量, 先利用巨正则系综蒙特卡罗方法 (grand canonical Monte Carlo, GCMC $)^{[19,21]}$ 确定吸附等温线. 然后使用 NVT 系综下的构型偏倚蒙特卡罗 (configurational bias Monte Carlo, CBMC) ${ }^{[22-23]}$ 模拟方法获得初始构 型. 在动力学运算中, 每个体系的运算步数为 $3 \times 10^{6}$ 步, 其中前 $1.5 \times 10^{6}$ 步用于使体系达到平衡, 后 $1.5 \times$ $10^{6}$ 步用于抽样. 时间步长为 $1 \mathrm{fs}$, 并且每隔 $0.1 \mathrm{ps}$ 保存一个体系构型, 这样各体系均保存了 15000 个 平衡态下的构型样本用于分析研究. 其中自扩散系 数 $D_{\mathrm{s}}$ 使用粒子均方位移法 (mean-square displacements, MSD)计算 ${ }^{[11,24]}$, 质心分布图使用XcrysDen 软 件 ${ }^{[25]}$ 绘制.

\section{2 结果与讨论}

\section{1 力场验证}

为确认力场的可靠性, 首先对气体 $\mathrm{Ar}$ 在 $\mathrm{Cu}-$ BTC 中的扩散行为进行了模拟. Ar 使用与文献[5] 相同的模型来描述, 模拟结果见图 5. 从图中可以看

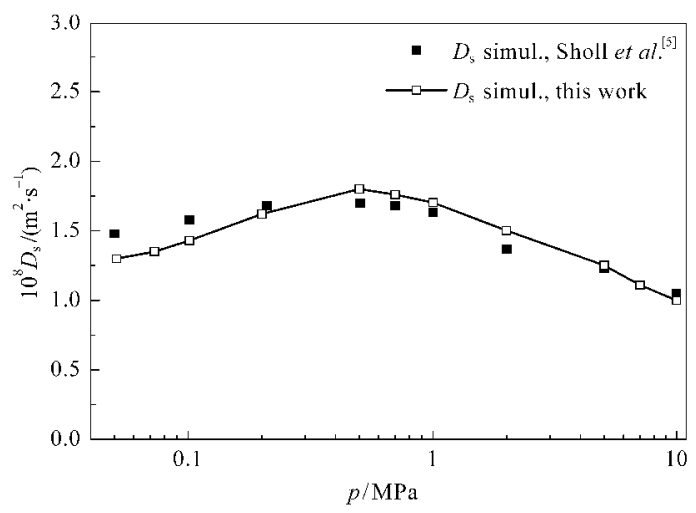

图 $5298 \mathrm{~K}$ 下 Ar 气体在 Cu-BTC 中的自扩散系数与 文献值比较

Fig.5 Comparison of the simulated self-diffusion coefficients of $\mathrm{Ar}$ in $\mathrm{Cu}-\mathrm{BTC}$ at $298 \mathrm{~K}$ with the values in literature
出模拟结果与文献[5]中的结果吻合较好. 这说明 $\mathrm{UFF}$ 力场可以应用于气体在含有孔笼和正交孔道 的 MOFs 中扩散行为的动力学研究. 而用 TraPPE 力场来描述甲烷分子在材料中的扩散, 也已被证明 是合适的 ${ }^{[5,19}$. 因此, 使用这一套力场来研究甲烷的 扩散行为是可行的.

\section{2 吸附等温线}

为对不同压力下甲烷气体在材料中的扩散性质 进行研究, 首先模拟了不同压力下甲烷气体在材料 中的吸附等温线, 如图 6 所示. P-C 材料用实心图形 表示, IRMOF 系列材料用空心图形标示. 从图中可 以看出, 在较高压力下, 由于 $\mathrm{Cu}-\mathrm{BTC}$ 的晶胞参数、 孔笼和正交孔道都比较小, 所以每个单元晶胞内吸 附甲烷分子数相对较小. 而 PCN-6', mesoMOF-1 和

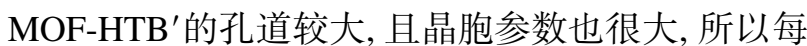
个晶胞内, 甲烷的吸附量明显高于其他几种材料. 由 于 IRMOF-11 和 IRMOF-13 两种为连锁结构材料, 其孔道被切割成复杂的小区域, 产生了额外的小孔 径复杂孔道. 这种复杂的小孔结构有利于气体分子 的吸附 ${ }^{[26]}$, 所以在低压情况下吸附量略微高于其他 六种材料, 而在较高压力下, 吸附量相对其他材料 小. 虽然这种细小而复杂的小孔结构在中低压下有 利于气体分子的吸附, 但同样也减小了气体分子在 孔道中运动的空间, 不利于甲烷分子在其中的扩散. 因此, 接下来我们将结合不同压力下的吸附量来对 气体分子的扩散进行研究.

\section{3 自扩散系数}

甲烷在各种材料中的自扩散系数如图 7 所示, 其中 IRMOFs 中的自扩散系数取自本组以前的工 作 ${ }^{[2]}$. 从图中可以看出, 甲烷在各种材料中自扩散系 数都会随压力的增加而增加, 达到一个极大值后开

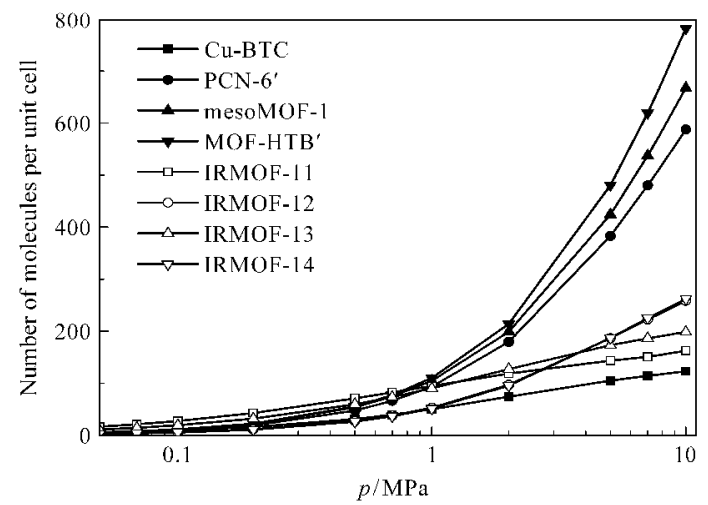

图 $6298 \mathrm{~K}$ 下甲烷气体在各种材料中的吸附等温线

Fig.6 Adsorption isotherms of methane in different materials at $298 \mathrm{~K}$ 


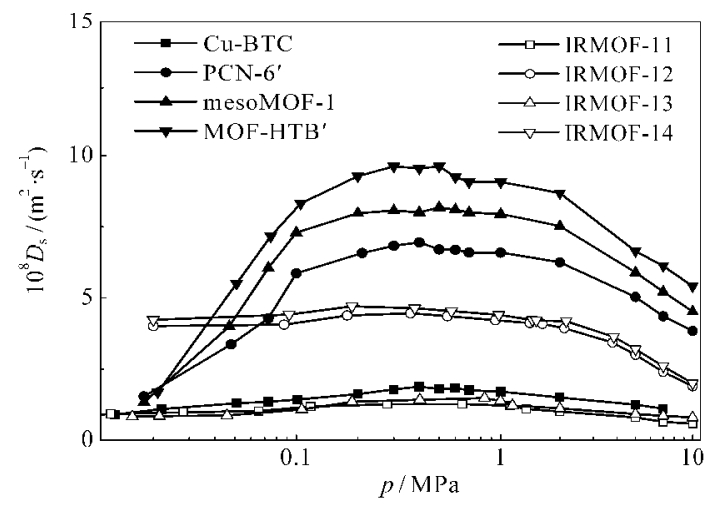

图 $7298 \mathrm{~K}$ 下甲烷气体在各种材料中的自扩散系数

Fig.7 Self-diffusion coefficients of methane in different materials at $298 \mathrm{~K}$

始降低. 这是由于材料中不同位置对气体的吸附能 力强弱不同导致的. 在压力很低时(大体上小于 0.3 $\mathrm{MPa}$ ), 气体分子优先吸附于较强的吸附位点, 且吸 附的分子数较少. 由于被牢固地吸附, 气体分子自扩 散系数较小. 随着压力的增加, 被吸附的分子数增 加, 同时 MOF 材料骨架上的优先吸附位置逐渐被 覆盖, 这使得甲烷分子的扩散速度加快, 自扩散系数 升高. 而随着压力的进一步增加(大体上大于 1.0 $\mathrm{MPa}$ ), 骨架中吸附的气体分子逐渐增多, 分子之间 的相互碰撞也随之增多, 进而阻碍其运动, 因此, 此 时气体的自扩散系数开始减小.

两类材料中甲烷的自扩散系数在中低压下均随 压力的增加而增加, 在 P-C 材料中比较明显, 这是 由于在 P-C 材料中存在两类结构不同的孔道, 分子 在其中的扩散速率差别很大, 而在 IRMOFs 中孔道 结构单一, 气体分子扩散速率变化较小, 曲线都较平 缓 (见图 7). 为了进一步比较甲烷在两类不同结构的 材料中自扩散系数的差异, 我们计算了极低压力下 和自扩散系数达到最大值情况下每种材料中气体自 扩散系数的绝对差和相对比例, 如图 8 所示, 其中 $D_{\mathrm{sPP}}$ 表示极低压力情况下 (模拟中材料只吸附一个 甲烷分子时的压力)的自扩散系数, $D_{\mathrm{s} \text { max }}$ 表示整个压 力区间内 (本文中为 0.01-3.00 MPa) 自扩散系数的 最大值. 从图中可以看出, 对极低压力情况下自扩散 系数与模拟压力区间内自扩散系数的最大值进行比 较, 不论是绝对差值(图 8A)还是相对比例(图 8B) P-C 材料中甲烷自扩散系数变化明显, 而在 IRMOF 材料中变化很小. 结合甲烷在各材料中的自扩散系 数随压力变化图(图 7)可以看出, 气体分子在 P-C 材 料中的自扩散系数受压力影响较大, 因此可以通过
改变外界压力来控制气体分子在此类材料中的扩散 速率.

\section{4 质心分布}

为深人理解甲烷分子在不同结构 MOF 材料中 的扩散差异, 我们进一步研究了甲烷在材料中的质 心分布. 吸附分子在材料中的质心分布图由对所有 构形样本在其平衡阶段进行统计叠加绘制而成, 以 分子密度的形式表现材料某一层面上各个位置气体 分子出现的概率. 在本工作中选取整个单元晶胞内 气体分子进行统计叠加, 用来描述气体分子在整个 单元晶胞内的自扩散行为.

为讨论自扩散系数随压力的变化与材料中气体 运动规律之间的关系, 根据吸附等温线(图 6)和自扩 散系数随压力的变化(图 7)选取三个压力点下材料 中甲烷分子的质心分布图进行研究: (1)材料中刚开
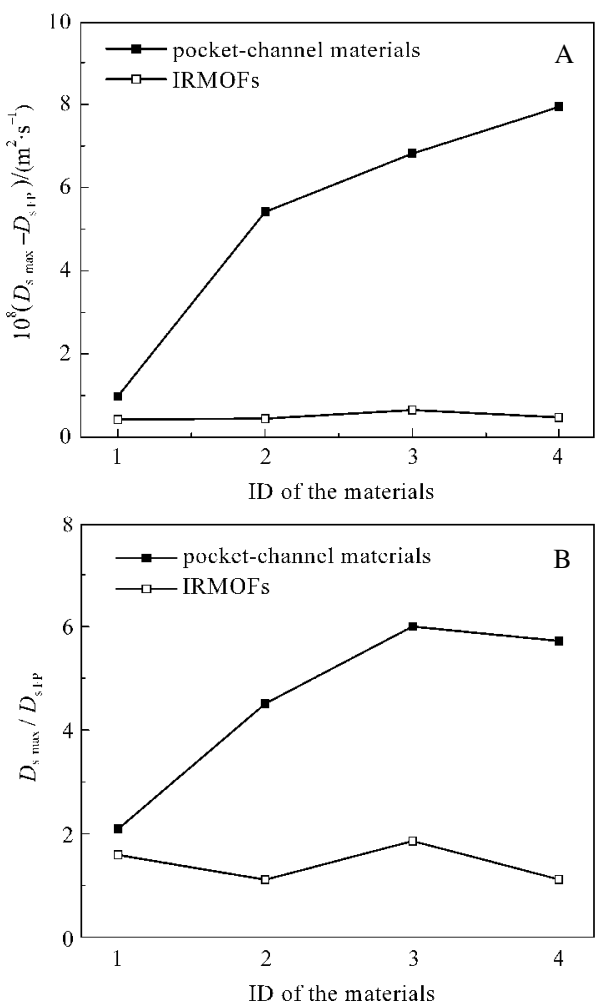

图 $8298 \mathrm{~K}$ 下 pocket-channel 材料与 IRMOF 材料中极 低压力情况下自扩散系数与模拟压力区间内自扩散系数的 最大值之间的比较

Fig.8 Comparison of self-diffusion coefficient of methane in pocket-channel materials and IRMOFs between the diffusion at very low pressure and maximal diffusion rate point at $298 \mathrm{~K}$

(A) absolute difference, (B) relative difference; For P-C materials,

IDs of Cu-BTC, PCN-6' ${ }^{\prime}$, mesoMOF-1, MOF-HTB' are 1, 2, 3, 4, respectively; for IRMOFs, IDs of IRMOF-11, IRMOF-12, IRMOF-13, IRMOF-14 are 1, 2, 3, 4, respectively. 


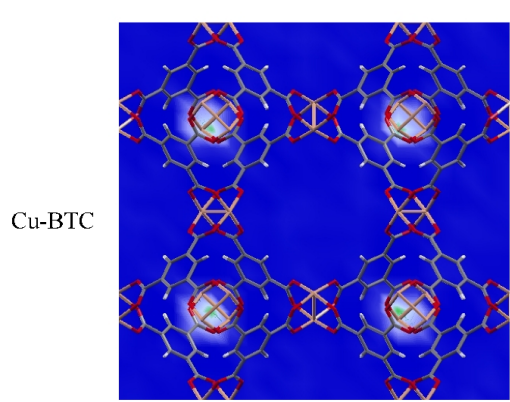

$0.02 \mathrm{MPa}$

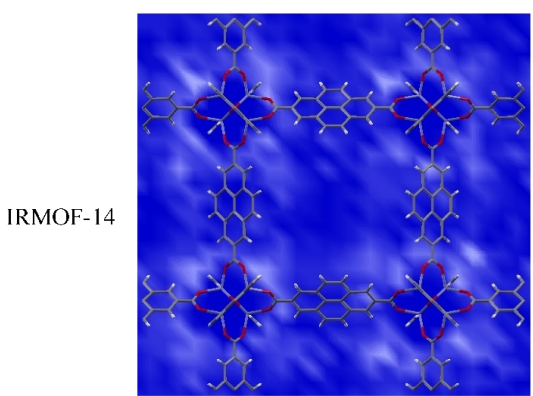

$0.02 \mathrm{MPa}$

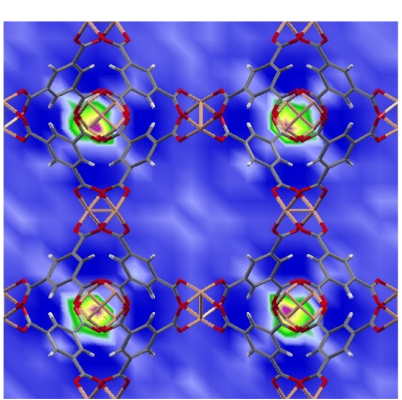

$0.2 \mathrm{MPa}$

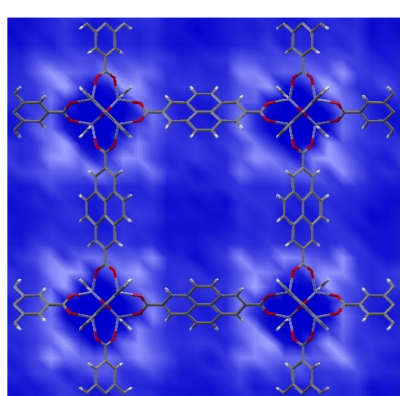

$0.2 \mathrm{MPa}$

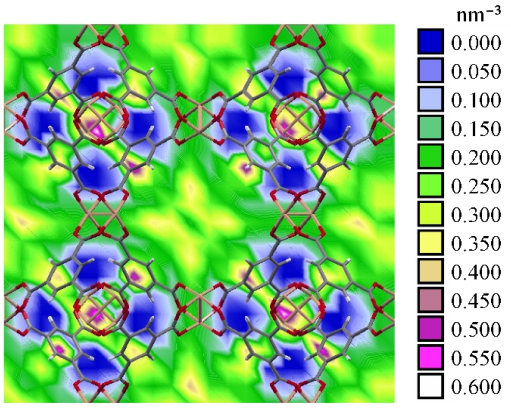

$2.0 \mathrm{MPa}$

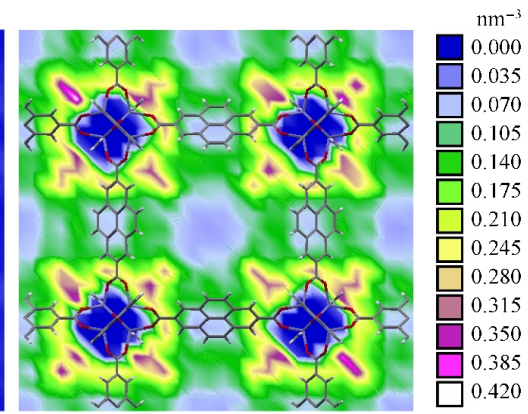

$2.0 \mathrm{MPa}$

图 $9298 \mathrm{~K}$ 下 Cu-BTC 和 IRMOF-14 中甲烷在晶胞内 $y-z$ 平面质心分布图

Fig.9 Contour plots of the center of mass (COM) probability densities of methane in the $y-z$ plane for all of the methane molecules

始出现气体分子 $(0.02 \mathrm{MPa}),(2)$ 材料中气体分子自 扩散系数接近最大值 $(0.2 \mathrm{MPa}),(3)$ 材料中气体分子 自扩散系数随着压力增加开始下降 $(2.0 \mathrm{MPa})$. 由于 同系列材料得到的结果基本类似, 因此以 $\mathrm{Cu}-\mathrm{BTC}$ 和 IRMOF-14 为例研究甲烷在晶胞内 $y-z$ 平面质心 分布图, 从这张图中可以直观观察吸附于材料内甲 烷分子出现的位置. 如图 9 所示, 在 $0.02 \mathrm{MPa}$ 下, 由 于两类材料孔道结构上的差异, 被吸附气体分子运 动的区域也存在差异. P-C 材料中孔笼区域是气体 的优先吸附位置, 在低压下气体分子大部分集中在 这一区域. 在 $\mathrm{Cu}-\mathrm{BTC}$ 中, 大部分甲烷分子都集中吸 附在 linker 组成的孔笼中. P-C 材料的孔笼相对较 小, 由于空间位阻效应阻碍了分子在这一区域的运 动, 所以低压下气体在 $\mathrm{Cu}-\mathrm{BTC}$ 中自扩散系数都很 小, 自扩散速率在 $0.9 \times 10^{-8}-1.4 \times 10^{-8} \mathrm{~m}^{2} \cdot \mathrm{s}^{-1}$ 之间, 这 与连锁结构的 IRMOF 材料中的情况相似, 由于连 锁结构, 材料孔道被切割成复杂的小区域. 这种复杂 小孔结构有利于气体分子的吸附 ${ }^{[26]}$, 同时也对气体 分子的扩散不利. 所以在低压下 P-C 材料中气体分 子自扩散速率与连锁结构的 IRMOF 材料中相当. 而 IRMOF-14 中甲烷分子优先吸附在金属簇附 近 ${ }^{[26}$, 但由于其具有较好的孔道连通性, 因此可观测
到甲烷分子较平均地分布在整个孔道之内. 随着压 力的增加, 在 $0.2 \mathrm{MPa}$ 下, 甲烷分子吸附量增加, 优 先吸附位点都接近或超过饱和, 这使甲烷分子向材 料中其他位置运动. 由于 IRMOF 材料中孔道结构 单一, 所以在 IRMOFs 中甲烷分子较平均地分布在 整个孔道之内, 空间约束对自扩散系数基本无影响. 这个压力下, 甲烷分子的自扩散系数与低压 $(0.02$ $\mathrm{MPa})$ 下基本相同. 在P-C材料中, 随着压力的增加, P-C 材料中气体分子逐渐进入较大正交孔道结构 中, 空间位阻的效果降低, 分子运动阻碍减小, 这使 在这一压力区间 (对 $298 \mathrm{~K}$ 下 P-C 材料中甲烷气体 来讲该压力范围约为 0.02-0.4 MPa), 气体分子的自 扩散系数随压力增加而明显增加, 而 $0.2 \mathrm{MPa}$ 正在 此压力范围之内, 故气体分子的自扩散系数较低压 (0.02 MPa)下有明显增加(图 7). 在 $2.0 \mathrm{MPa}$ 下, 甲烷 分子在两类材料中都接近饱和, 气体分子在孔道各 处均大量出现, 气体分子与气体分子之间的碰撞明 显阻碍了气体分子本身的扩散, 所以在此情况下分 子在两种材料中的自扩散系数都开始随压力的增 高而降低(图 7). 由此可见, 选择具有适当孔道结构 的 MOF 材料(如 P-C 材料), 以及适当的压力范围, 可以很好地控制气体分子在其中的扩散速率. 


\section{3 结 论}

通过分子动力学方法, 结合质心分布图, 以甲烷 为探针分子研究了两类孔道具有代表性的 MOF 材 料中孔道结构对气体扩散速率的影响. 模拟结果表 明, IRMOFs 中的气体分子主要吸附在三维正交孔 道中金属簇附近区域, 增加压力后可向正交孔道扩 散; 由于良好的孔道连通性, 气体分子自扩散系数 随压力变化并不明显, 利用控制压力很难控制气体 扩散快慢. 而 P-C 材料中的气体分子在低压时吸附 在孔笼结构中, 随压力增加可向正交孔道扩散; 由 于扩散路径的改变, 气体分子自扩散速率随压力增 加变化明显, 气体分子自扩散的可控范围较大. 在中 低压下, linker 尺寸越大, 气体在其中的自扩散系数 随压力增加而增加越明显, 同时其自扩散系数最大 值也越大. 因此, 对具有 P-C 结构特性的 MOF 材料, 中低压下通过控制压力可以控制气体在其中的扩散 速率. 这为 $\mathrm{MOF}$ 材料在气体存储、分离等方面的实 际应用提供参考信息.

\section{References}

1 Férey, G. Chem. Soc. Rev., 2008, 37: 191

2 Talu, O.; Sun, M. S.; Shah, D. B. AIChE J., 1998, 44: 681

3 Sarkisov, L.; Düren, T.; Snurr, R. Q. Mol. Phys., 2004, 102: 211

4 Skoulidas, A. I. J. Am. Chem. Soc., 2004, 126: 1356

5 Skoulidas, A. I.; Sholl, D. S. J. Phys. Chem. B, 2005, 109: 15760

6 Liu, J. C.; Lee, J. Y.; Pan, L.; Obermyer, R. T.; Simizu, S.; Zande, B.; Li, J.; Sankar, S. G.; Johnson, J. K. J. Phys. Chem. C, 2008, 112: 2911
7 Zeng, Y. Y.; Zhang, B. J. Acta Phys. -Chim. Sin., 2008, 24: 1493 [曾余瑶, 张秉坚. 物理化学学报, 2008, 24: 1493]

8 Krishna, R.; van Baten, J. M. Chem. Eng. Sci., 2008, 63: 3120

9 Babarao, R.; Jiang, J. W. Langmuir, 2008, 24: 5474

10 Yang, Q. Y.; Zhong, C. L.; Chen, J. F. J. Phys. Chem. C, 2008, 112: 1562

11 Yang, Q. Y.; Zhong, C. L. J. Phys. Chem. B, 2005, 109: 11862

12 Liu, B.; Yang, Q. Y.; Xue, C. Y.; Zhong, C. L.; Smit, B. Phys. Chem. Chem. Phys., 2008, 10: 3244

13 Chui, S. S. Y.; Lo, S. M. F.; Charmant, J. P. H.; Orpen, A. G.; Williams, I. D. Science, 1999, 283: 1148

14 Ma, S. Q.; Sun, D. F.; Michael, A.; Jacqueline, A. F.; Sean, P.; Zhou, H. C. J. Am. Chem. Soc., 2007, 129: 1858

15 Wang, X. S.; Ma, S. Q.; Sun, D. F.; Sean, P.; Zhou, H. C. J. Am. Chem. Soc., 2006, 128: 16474

16 Eddaoudi, M.; Kim, J.; Rosi, N.; Vodak, D.; Wachter, J.; O'Keefe, M.; Yaghi, O. M. Science, 2002, 295: 469

17 Materials Studio Version 3.0. San Diego: Accelrys Inc., 2003

18 Martin, M. G.; Siepmann, J. I. J Phys. Chem. B, 1998, 102: 2569

19 Yang, Q. Y.; Zhong, C. L. J. Phys. Chem. B, 2006, 110: 17776

20 Keskin, S.; Sholl, D. S. J. Phys. Chem. C, 2007, 111: 14055

21 Yang, Q. Y.; Zhong, C. L. ChemPhysChem, 2006, 7: 1417

22 Vlugt, T. J. H.; Krishna, R.; Smit, B. J. Phys. Chem. B, 1999, 103: 1102

23 Smit, B.; Siepmann, J. I. J. Phys. Chem., 1994, 98: 8442

24 Frenkel, D.; Smit, B. Understanding molecular simulation: from algorithms to applications. San Diego: Academic Press, 2002: 63-108

25 Kokalj, A. Comput. Mater. Sci., 2003, 28: 155

26 Xue, C. Y.; Zhou, Z. E.; Yang, Q. Y.; Zhong, C. L. Chin. J. Chem. Eng., 2009, 17: 580 